\title{
Analysis of Medical Consultation Patterns in Medical and Surgical Intensive Care Units: Changes in the Pattern of Consultation after the Implementation of Intensivist-Directed Care
}

\author{
Min-Jung Bang a, So-Kyung Yoon a, Kyoung Won Yoon a, Eunmi Gil a,b, Keesang Yoo a, Kyoung Jin Choi a , \\ Chi-Min Park a,b,* \\ ${ }^{a}$ Department of Surgery, Samsung Medical Center, Sungkyunkwan University School of Medicine, Seoul, Korea \\ ${ }^{b}$ Department of Critical Care Medicine, Samsung Medical Center, Sungkyunkwan University School of Medicine, Seoul, Korea
}

\section{Article history:}

Recevied: October 23, 2020

Revised: November 8, 2020

Accepted: November 10, 2020

\section{${ }^{*}$ Corresponding Author:}

Chi-Min Park

Department of Surgery and Critical Care Medicine, Samsung Medical Center Sungkyunkwan University School of

Medicine, 81 Irwon-ro, Gangnam-gu, Seoul

06351, Korea

E-mail: dr99.park@samsung.com

\section{ORCID}

Min-Jung Bang

https://orcid.org/0000-0003-3536-7526

So-Kyoung Yoon

https://orcid.org/0000-0001-6004-664X

Kyoung Won Yoon

https://orcid.org/0000-0002-9829-5024

Eunmi Gil

https://orcid.org/0000-0002-2002-0936

Keesang Yoo

https://orcid.org/0000-0001-6476-8471

Kyoung Jin Choi

https://orcid.org/0000-0003-2639-2594

Chi-Min Park

https://orcid.org/0000-0002-8496-3546

\section{ABSTRACT}

Purpose: Critically ill patients often require multidisciplinary treatment for both acute illnesses and pre-existing medical conditions. Since different medical conditions are managed in the intensive care unit (ICU), consultation is often required. This study aimed to identify the frequency and type of consultation required and analyze changes in consultation patterns after the introduction of intensivist-directed care in the surgical ICU (SICU).

Methods: Between June 2006 and December 2013, a retrospective cohort study was conducted to identify the frequency and type of consultation at 3 different ICUs. Consultations for patients who were admitted to the ICUs for more than 48 consecutive hours were included. The pattern of consultations in each ICU was investigated. In addition, the pattern of consultations before and after the implementation of intensivist-directed care in the SICU was compared.

Results: During the study, 11,053 consultations were requested for 7,774 critically ill patients in a total of 3 ICUs. Consultations with the Departments of Cardiology, Infectious Diseases, and Pulmonology were requested most frequently in the SICU. However, after the implementation of the intensivist-directed care approach, there was an increase in the frequency of consultation requests to the Department of Neurology, followed by the Departments of Cardiology, and Infectious Diseases.

Conclusion: Analysis of consultation patterns is an important method of assessing the complexity and severity of illnesses, and of evaluating the needs of available health system resources. Based on our findings, we suggest the development of an appropriate protocol for frequently consulted services.

Keywords: consultation, critical care, critical illness, intensive care units, physician

\section{Introduction}

Caring for critically ill patients in the intensive care unit (ICU) setting is complex, and often requires a high level of expertise and a multidisciplinary team of healthcare professionals [1-3]. Since a multidisciplinary approach is required, intensivists serve as supervisors overseeing all systems [4]. These intensivists have the multidisciplinary expertise necessary for the treatment of critical illnesses, but the advice of specialists in particular fields is also essential [58]. Therefore, accessibility to specialists should be made more available through the appointment of ICU-dedicated physicians or through exclusive routes. In addition, considering that most consultations in the ICU are urgent, a way to respond in a timelier manner needs to be ascertained, rather than being processed through the general ward.

Inpatient hospital consultation is a useful area for exploration as it determines the quality of care and resource utilization 
in the ICU [9]. However, there are few studies on consultation patterns in the ICU, especially in the surgical ICU (SICU). In the past, many ICUs used the open ICU model in which patients were cared for by their primary care physician [10]. Physicians addressed complex problems by referring patients to specialist consultations in other departments [11,12]. With the introduction of an intensivist who is capable of providing comprehensive care, the frequency of consultations is expected to reduce. However, there is still an increased need for consultations with specialists for patients with complex medical needs in the ICU, often requiring support from a wide variety of providers; thus, the inpatient hospital consultation remains an important practice to ensure the delivery of comprehensive and coordinated care.

The objective of this study was to compare the individual consultation patterns in different types of ICUs in an academic hospital, to provide information on the efficient utilization of hospital resources, and to determine whether the introduction of an intensivist affects the consultation frequency and patterns in the SICU. These findings may be used as evidence to develop effective frameworks and models for improving resource allocation in hospital settings.

\section{Materials and Methods}

\section{Study design}

This was a retrospective cohort study designed to identify the frequency and types of consultations in the medical and oncology ICUs and SICU in a single academic teaching hospital between June 2006 and December 2013. The Institutional Review Board approved the study protocol (IRB no.: 2020-09154-100).

Samsung Medical Center (SMC), located in Seoul, South Korea, serves as an academic teaching hospital with approximately 2,000 beds. The hospital has a range of departments: Emergency, Cardiology, Cardiac Surgery, Medical, Neurosurgery, Neuro-pediatric, Pediatric-Cardiac, Transplantation-Surgical, Neonatal, Oncology-Medical, Surgery, and Oncology-Thoracic. In this study, the focus was on Medical, Oncology, and SICUs. Specialized Cardiac, Neurology, and Pediatric ICUs were excluded.

The medical ICU (MICU) is a 16-bed unit that provides interdisciplinary care for patients with a variety of serious medical conditions in a technologically advanced environment. The oncology ICU (OICU) provides intensive monitoring and treatment to cancer patients with emergency symptoms. The OICU is a 14-bed unit that provides care to approximately 350 critically ill cancer patients per year. The SICU is a 13-bed unit. The patient population mainly comprises postoperative patients that are directly admitted to the ICU after scheduled or emergency surgery, including patients who experience surgical complications or other medical problems in the general medical wards or the emergency room. When the SICU was established in 2008, it used an open ICU model. Since April 2009, the SICU gradually converted to an intensivist-directed ICU managed by an intensivist who was board certified in both general surgery and critical care medicine [13].

\section{Study population}

The patients were aged 18 years or older who were admitted to the MICU, OICU, or SICU for intensive care for more than 48 hours between June 2006 and December 2013. In total, 7,754 patients were provided intensive care during the study period and 11,053 consultations occurred.

\section{Data collection}

In the SMC, consultations for inpatients in any medical subspecialties, can be offered promptly (within 24 hours). The intensive care physician requests a consultation with a specialist for the patient's problems using a computerized physician order entry. In ICUs, a consultation is usually requested after a team discussion or at the request of the intensivist. After collecting the information on the departments that in which a patient had been consulted, the data reflected how many departments gave consultations to a patient, rather than the number of consultations provided by each department. For example, even though 1 patient had 4 consultations with cardiology during their stay in ICU, it was calculated as 1 . Simple consultations generated for examinations, radiological procedures, nutrition support, pharmacy medications, and the approval of restricted antibiotics were excluded. Only the name of the departments that were involved in the consultations could be identified because this was a retrospective study; the details of the consultation were unknown (e.g., in the case of cardiologic problems, we could not investigate whether the cause was arrhythmia or vascular disease).

\section{Statistical analysis}

Consultation patterns and frequencies in each ICU were compared. Statistical analyses were performed using SAS Version 9.4 (SAS Institute, Cary, NC) and R 3.1.0 (Vienna, Austria). The Wilcoxon rank-sum test was used to analyze the pattern of consultation. A $p<0.05$ was considered statistically significant.

\section{Subgroup analysis}

In the SICU, consultations that occurred during 2 distinct phases were compared: Phase 1, between January 2008 and March 2009 before the implementation of the intensivist-directed ICU model, and Phase 2, between April 2009 and December 2013, after complete conversion to the intensivist-directed ICU model. 


\section{Results}

\section{Overall data}

Almost half of the patients underwent at least 1 consultation (48.3\%). In total, 7,774 patients were admitted to the ICU during the study period and a total of 11,053 consultations took place. The number of consultations that occurred for each patient ranged between $0-59$, with an average of 1.4 [ \pm 2.9 (SD)] consultations (Table 1 ).

Data on consultations with subspecialty physicians requested by the intensive care physician are shown in Figure 1A.

Physicians from the Departments of Infectious Diseases (19.6\%), Neurology (11.7\%), and Cardiology (10.7\%) were most frequently consulted.

The frequencies of consultation in the MICU, OICU, and SICU were analyzed (Figure 1B). Regarding the MICU, a total of 3,172 patients were reviewed in the study and 5,354 consultations $(1.7 \pm 2.9)$ took place. Figure $1 \mathrm{~B}$ shows the consultation frequencies: physicians from the Department of Infectious Disease were the most frequently consulted (17.1\%), followed by those from the Departments of Neurology (13.5\%), and Cardiology (9.7\%). Regarding the OICU, a total of 2,324 consultations were made for 2,384 patients, with an average of $1.4( \pm 2.7)$ consultations per patient. Figure $1 \mathrm{~B}$ shows that physicians from the Departments of Infectious Diseases (27.1\%), Neurology (12.1\%), and Cardiology (8.7\%) were the most frequently consulted. For the SICU, a total of 2,198 patients were reviewed in the study, and 2,452 consultations $(1.1 \pm 2.7)$ took place. The consultation frequencies in the SICU were as follows: physicians from the Department of Cardiology were the most frequently consulted (15.5\%), followed by those from the Departments of Infectious Diseases (15.3\%), and Pulmonology (8.9\%; Figure 1B).

Table 1. Overall prevalence of patient consultations in ICU.

\begin{tabular}{lccccc}
\hline & & & & \multicolumn{2}{c}{ Prevalence of consultation } \\
\cline { 3 - 6 } Overall data & Patients & Consultations & Mean \pm SD & Median \pm IQR & $0.0 \pm 2.0$ \\
\hline Total & 7,754 & 11,053 & $1.4 \pm 2.9$ & $0.0 \pm 2.0$ & $0-41$ \\
Medical ICU & 3,172 & 5,354 & $1.7 \pm 3.0$ & $0.0 \pm 2.0$ & $0-59$ \\
Oncologic ICU & 2,384 & 3,274 & $1.4 \pm 1.7$ & $0.0 \pm 1.0$ & $0-31$ \\
Surgical ICU & 2,198 & 2,452 & $1.1 \pm 2.7$ & 0 & 0.0 \\
\hline
\end{tabular}

ICU = intensive care unit; IQR = interquartile range.

(A)

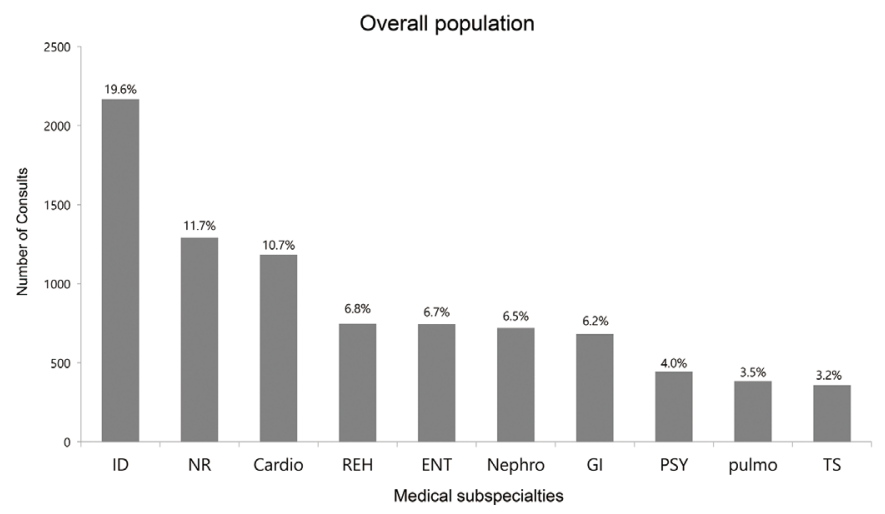

(B)

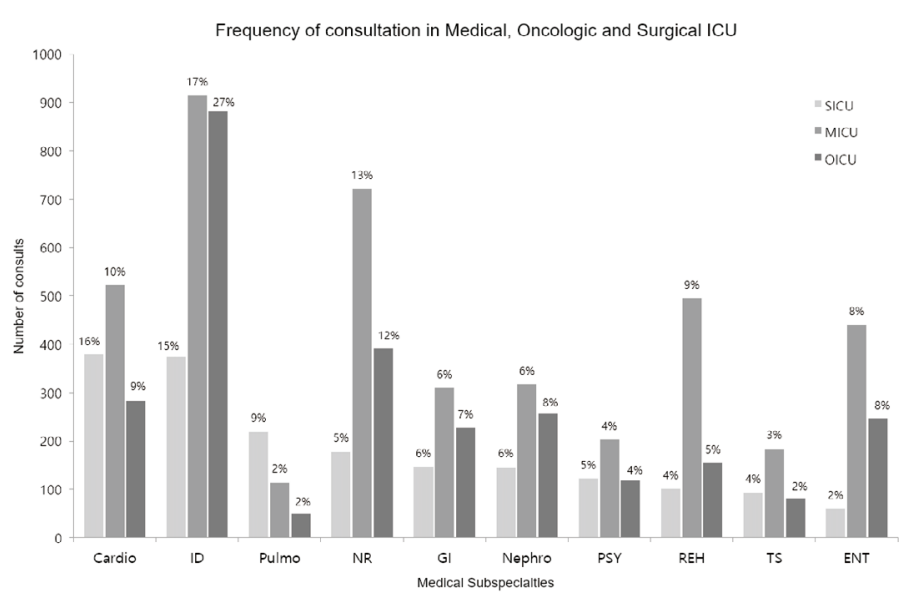

Figure 1. Number of consultations by medical subspecialities. (A) Distribution of subspecialty consultations requested by intensive care physicians. (B) Frequency of consultations with the Departments of Cardiology (15.5\%), Infectious Diseases (15.3\%), and Pulmonology (8.9\%) in the SICU. Frequency of consultations with the Departments of Infectious Diseases (17.1\%), Neurology (13.5\%), and Cardiology (9.7\%) in the medical ICU. And frequency of consultations with the Departments of Infectious Diseases (27.1\%), Neurology (12.1\%), and Cardiology (8.7\%) in the oncology ICU. Cardio = cardiology; ENT = otolaryngology; GI = gastroenterology; ICU = intensive care unit; ID = infectious disease; Nephro = nephrology; NR = neurology; PSY = psychiatry; Pulmo = pulmonology; REH = rehabilitation medicine; TS = thoracic surgery. 


\section{Changes in consultation trends before and after the adop- tion of the intensivist model in the SICU}

Since April 2009, the SICU has operated under the intensivist model. Figure 2 compares the consultation frequency before and after the implementation of intensivist-directed care in the SICU. On average, 1.12 consultations were made per patient; these consultations often took place with the Department of Infectious Diseases. The frequency of consultations decreased from 1.30 before the adoption of the intensivist model, to 1.09 whilst operating under the intensivist model, but this difference was not statistically significant $(p=0.53)$. After adopting the intensivist-directed care model at the SICU, the frequency of consultations given by each department changed. A decrease in frequency of consultations given by the Department of Pulmonology (13.0\% vs. $7.5 \%$ ), and an increase in frequency in orthopedics (3.0\% vs. $6.5 \%$ ), rehabilitation $(3.0 \%$ vs. $4.5 \%$ ), and psychiatry (3.0\% vs. $6.0 \%$ ) were observed, however, these changes were not significant.

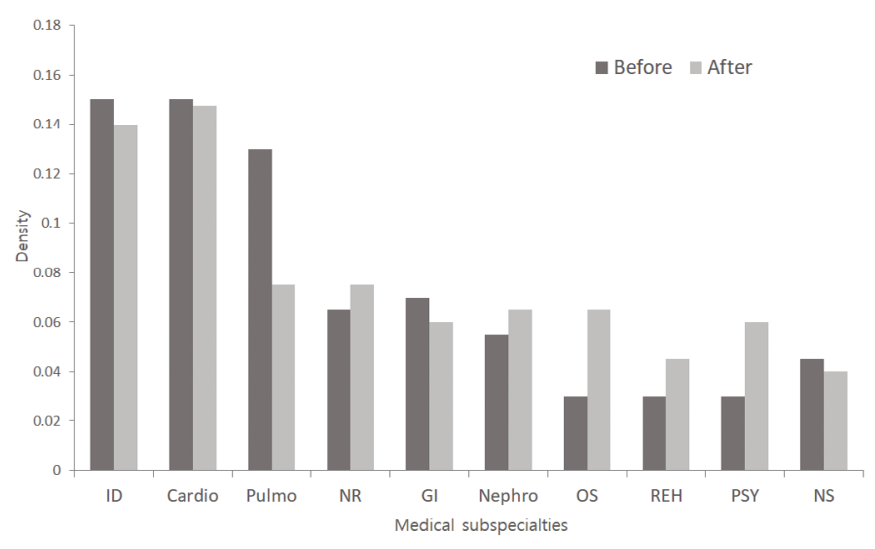

Figure 2. Comparison of the distribution of subspecialty consultations before and after the implementation of the intensivist model in the SICU. Note the decrease in frequency, pulmonology (13.0\% vs. $7.5 \%)$; increase in frequency, orthopedics (3.0\% vs. 6.5\%), rehabilitation $(3.0 \%$ vs. $4.5 \%$ ), and psychiatry (3.0\% vs. $6.0 \% ; p=0.53$ ). Distribution of consultation frequency (density) stratified by medical subspecialties. Before (black bar) is before adopting the intensivist model in the SICU, after (grey bar) is after adopting the intensivist model in the SICU. SICU = surgical intensive care unit.

\section{Discussion}

Critically ill patients often require multidisciplinary treatment approaches [1-3]. Consultation is 1 of the strategies to enhance the outcomes of ICU patients, and it is an essential indicator of patient complexity, and use of health resources [9]. However, consultation patterns in the patient population of a particular type of ICU have not been explored. This study analyzed the consultation patterns in 3 specific ICUs, and compared the individual consultation patterns that occurred in different types of ICUs in a single academic teaching hospital over an 8-year period. The changes in consultation patterns were also investigated in the periods before and after the SICU came under the direction of a surgical intensivist certified in both general surgery and critical care medicine.

The key finding of this study was that in all populations, the highest frequency of consultations according to department, was by Infectious Diseases, followed by Neurology, and Cardiology. Care provided by Infectious Disease was the most heavily utilized specialty care in the ICU patient population overall. Other studies have reported similar findings [9], possibly because the incidence of nosocomial infections in ICUs is about 2-5 times higher compared with inpatients in General hospital wards [14]. More than half of ICU patients received at least 1 course of antibiotics during their stay in the ICU $[15,16]$, and it is well documented that the presence of an infection is a key determinant of an adverse outcome in critically ill patients $[16,17]$. Intensivists know the importance of infection control and have basic plans for early treatment. However, there are many situations in the ICU that require the use of advanced therapeutic antibiotics. Recognizing this concept, a dedicated infectious disease physician was assigned to the SICU in 2015. Since then, it has become necessary to collect and confirm objective data. Thus, accessibility to specialists is expected to improve; dedicated physicians will be aware of the patient's condition and a constant line of care will be maintained, resulting in a substantial decrease in length of stay in hospital and inpatient mortality [18]. Therefore, infectious disease specialists play increasingly important roles dedicated in the management of antibiotics, given the increased incidence of diseases caused by multidrug-resistant bacteria in recent years.

Unlike the patterns in other ICUs, the frequently consulted departments in the SICU was highest for the Department of Cardiology, followed by Infectious Diseases, and Neurology. This pattern is thought to be due to the rapid turnover of the patient population in the SICU, which is used for postoperative care. Patients admitted to the ICU after surgery often had heart problems, identified on preoperative examinations. This led to a high risk of heart problems after surgery and a higher frequency of consultation with the Cardiology department than with the Infectious Diseases department [19,20].

After adopting the intensivist model in the SICU, the frequency of pulmonology consultations decreased because of the involvement of the intensivist, who was relatively alert to issues related to the respiratory system compared to the surgeon. The intensivist has better contact with the patients compared to the surgeons who spend most of their day in the operating room. In contrast, the frequencies of consultations with the Department of Psychiatry, and Rehabilitation increased. We suspect that this pattern was due to the involvement of the ICU intensivist, who is aware of the latest research. Recently, the importance of managing delirium and early rehabilitation 
in ICU patients has been reported [21].

The frequency of consultations with the Department of Orthopedics increased after the introduction of an intensivist to the SICU, possibly because this step resulted in the management of multiple trauma patients with orthopedic problems in the SICU.

At the beginning of the study, it was expected that the frequency of consultations in the SICU would decrease after the introduction of an intensivist capable of providing general comprehensive care, but the change in consultation frequency was statistically insignificant. These results suggest that although the introduction of intensivists may change the consultation pattern for heavily utilized departments, the demand for assistance from other physicians through consultation is still high, given the changing trends in ICU settings, and requirement of multidisciplinary management for ICU patients.

The ICU is a highly specified and sophisticated area of a hospital that is specifically designed, staffed, located, furnished and equipped, and dedicated to the management of critically ill patients. The innate complexity of the ICU makes systematic organizational structures necessary. To establish a systematic structure, epidemiological factors such as patient characteristics, diagnostic methods, and system-enabled patterns need to be assessed because all these factors play an important role in the ICU. The consultation patterns observed in this study will be a guide for the implementation of future systems regarding which aspect of the ICU should be observed closely, such as multidisciplinary consultation. This would result in a consultation pattern different from the consultation pattern observed in a semi-closed or closed ICU. In this study, only the frequency of consultation to each department was investigated. Had the contents of each consultation been analyzed, more would have been discovered about what kind of support and consultation system is needed. In future, research into the content of consultations by each department needs to be explored.

This study is subject to a number of limitations. Firstly, its retrospective design may have predisposed it to selection bias. Secondly, the results of this study cannot be generalized to all hospitals because it was the analysis of the patterns of consultation in ICUs of a single hospital. The application of the consultation pattern to all hospitals is limited because the accessibility of each department is different depending on the systems used in each country, and each hospital. Thirdly, the analysis only included the names of the departments involved in the consultations.

In conclusion, differences in consultation patterns were observed between ICUs because each ICU has a unique character. Furthermore, there were differences in consultation patterns in the SICU before and after the introduction of the intensivist system. This study used a retrospective approach to investigate the consultation frequencies and patterns in the ICU. Based on the findings, we suggest that an efficient consultation system is developed involving high-demand consulting departments, such as the Departments of Infectious Diseases, Neurology, and Cardiology, that can help ensure that these services are available and accessible. Furthermore, developing an appropriate protocol for frequently consulted services would address complex healthcare needs and improve clinical practice and quality of care for patients.

\section{Conflicts of Interest}

Chi-Min Park has been the editor in chief of Journal of Acute Care Surgery since January 2011, but had no role in the decision to publish this original article. No other potential conflict of interest relevant to this article was reported.

\section{Ethical Statement}

This research did not involve any human or animal experiment.

\section{References}

[1] Task Force of the American College of Critical Care Medicine Society of Critical Care Medicine. Guidelines for intensive care unit admission, discharge, and triage. Task Force of the American College of Critical Care Medicine, Society of Critical Care Medicine. Crit Care Med 1999;27(3):633-8.

[2] Hong KS. Intensivist as a Surgeon: The Role of a Surgeon in Critical Care Medicine. Ewha Med J 2017;40(2):61-5.

[3] Kim MM, Barnato AE, Angus DC, Fleisher LA, Kahn JM. The effect of multidisciplinary care teams on intensive care unit mortality. Arch Intern Med 2010;170(4):369-76.

[4] Dunn W, Murphy J. Should intensive care medicine itself be on the critical list? Chest 2009;135(4):892-4.

[5] Vincent JL. Critical care--where have we been and where are we going? Crit Care 2013;17 Suppl 1(Suppl 1):S2.

[6] Schweickert WD, Pohlman MC, Pohlman AS, Nigos C, Pawlik AJ, Esbrook $\mathrm{CL}$, et al. Early physical and occupational therapy in mechanically ventilated, critically ill patients: A randomised controlled trial. Lancet 2009;373(9678):1874-82.

[7] Lee TH. Proving and improving the value of consultations. Am J Med 2002;113(6):527-8.

[8] Raineri E, Pan A, Mondello P, Acquarolo A, Candiani A, Crema L. Role of the infectious diseases specialist consultant on the appropriateness of antimicrobial therapy prescription in an intensive care unit. Am J Infect Control 2008;36(4):283-90.

[9] Jordan MR, Conley J, Ghali WA. Consultation patterns and clinical correlates of consultation in a tertiary care setting. BMC Res Notes 2008;1:96.

[10] Ghorra S, Reinert SE, Cioffi W, Buczko G, Simms HH. Analysis of the effect of conversion from open to closed surgical intensive care unit. Ann Surg 1999;229(2):163-71.

[11] Tisherman SA, Kaplan L, Gracias VH, Beilman GJ, Toevs C, Byrnes MC, et al. Providing care for critically ill surgical patients: Challenges and recommendations. JAMA Surg 2013;148(7):669-74.

[12] Penkoske PA, Buchman TG. The relationship between the surgeon and 
the intensivist in the surgical intensive care unit. Surg Clin North Am 2006;86(6):1351-7.

[13] Park CM, Chun HK, Lee DS, Jeon K, Suh GY, Jeong JC. Impact of a surgical intensivist on the clinical outcomes of patients admitted to a surgical intensive care unit. Ann Surg Treat Res 2014;86(6):319-24.

[14] Dasgupta S, Das S, Chawan NS, Hazra A. Nosocomial infections in the intensive care unit: Incidence, risk factors, outcome and associated pathogens in a public tertiary teaching hospital of Eastern India. Indian J Crit Care Med 2015;19(1):14-20.

[15] Warren MM, Gibb AP, Walsh TS. Antibiotic prescription practice in an intensive care unit using twice-weekly collection of screening specimens: A prospective audit in a large UK teaching hospital. J Hosp Infect 2005;59(2):90-5.

[16] Kadri SS, Rhee C, Fortna GS, O'Grady NP. Critical Care Medicine and Infectious Diseases: An Emerging Combined Subspecialty in the United
States. Clin Infect Dis 2015;61(4):609-14.

[17] Vincent JL, Rello J, Marshall J, Silva E, Anzueto A, Martin CD, et al. International study of the prevalence and outcomes of infection in intensive care units. JAMA 2009;302(21):2323-9.

[18] Butt AA, Al Kaabi N, Saifuddin M, Krishnanreddy KM, Khan M, Jasim WH, et al. Impact of Infectious Diseases Team Consultation on Antimicrobial Use, Length of Stay and Mortality. Am J Med Sci 2015;350(3):191-4.

[19] Mangano DT. Perioperative cardiac morbidity. Anesthesiology 1990;72(1):153-84

[20] Maia PC, Abelha FJ. Predictors of major postoperative cardiac complications in a surgical ICU. Rev Port Cardiol 2008;27(3):321-8.

[21] Jakobson T, Karjagin J, Vipp L, Padar M, Parik AH, Starkopf L, et al. Postoperative complications and mortality after major gastrointestinal surgery. Medicina (Kaunas) 2014;50(2):111-7. 